\section{Canopy Position, Defoliation, and Girdling Influence Apple Nectar Production}

\author{
Richard J. Campbell', Richard D. Fell ${ }^{2}$, and Richard P. Marini \\ Department of Horticulture, Virginia Polytechnic Institute and State \\ University, Blacksburg, VA 24061
}

Additional index words. Malus domestics, high-performance thin-layer chromatography, flowering, sugar concentration

\begin{abstract}
Flowering spurs located at interior and exterior canopy positions of 'Stayman' and 'Delicious' apple (Malus domestics Borkh.) trees were girdled and/or defoliated to determine the influence on nectar production and composition. Nectar volume was less at exterior than interior canopy positions for 'Delicious', but not for 'Stayman'. Girdling suppressed nectar production by $92 \%$ and reduced the sugar concentration of the remaining nectar. Defoliation of nongirdled spurs had no effect on nectar sugar concentration, but defoliation of girdled spurs reduced nectar sugar concentration by $\mathbf{2 4 \%}$. Relative percentages of sucrose, glucose, and fructose, and the sucrose : hexose ratio were unaffected by any treatment. Nectar production of nongirdled spurs did not depend on the presence of spur leaves.
\end{abstract}

The shaded interior canopy of apple trees is generally less productive than the betterexposed outer canopy. The difference in productivity has been attributed in part to a reduction in flower bud formation (Jackson and Palmer, 1977) and fruitlet retention (Byers et al., 1990) due to reduced light levels and a subsequent reduction in assimilated carbohydrates available to the developing fruit. Nectar production and carbohydrate composition could also be influenced by carbohydrate availability within the canopy (Wykes, 1952), thereby influencing honeybee foraging behavior (Wailer, 1972; Wykes, 1952). Thus, pollination and fruitlet retention within the canopy may be affected. The objective of this study was to evaluate the influence of canopy position, defoliation, and girdling of flowering spurs on apple nectar production and composition.

All trees were located at the Virginia Polytechnic Institute and State Univ. Horticulture farm, Blacksburg. Five 23-year-old 'Stayman'/MM.11 trees were used in 1989 with a $2 \times 2$ factorial arrangement of interior/exterior canopy positions and foliated/ defoliated flowering spurs in a randomized complete block design (block $=$ tree). In 1990, five 15-year-old 'Red Prince Delicious'/MM. 111 trees were used with a $2 \times$ $2 \times 2$ factorial arrangement of interior/exterior positions, foliated/defoliated, and girdled/nongirdled flowering spurs. A single unbranched spur per treatment combination was selected within each tree based on uniformity of bud diameter and developmental stage. Individual spurs were defoliated and/

Received for publication 30 July 1990. The cost of publishing this paper was defrayed in part by the payment of page charges. Under postal regulations, this paper therefore must be hereby marked advertisement solely to indicate this fact.

'Dept. of Horticulture.

${ }^{2}$ Dept. of Entomology. or girdled at the tight cluster floral developmental stage and nectar samples were collected at full bloom (5 to 7 days later). Flowering spurs were covered with muslin bags 1 day before nectar collection to prevent honeybee visitation. Samples for sugar analysis were collected between 1100 and 1300 HR during periods of warm (> 20C), sunny weather by inserting a $1-\mu l$ microcapillary pipette into the nectary of a single flower (king bloom) and allowing the pipette to fill by capillary action. A single-flower sample was collected for sugar analysis for each treatment combination per tree.

Nectar volume was determined by measuring the portion of the 1- $\mu$ l pipette(s) filled with nectar. Samples were expelled into microcentrifuge tubes containing $200 \mu \mathrm{l}$ of $70 \%$ $(\mathrm{v} / \mathrm{v})$ ethanol and stored at $-22 \mathrm{C}$ until analysis. Qualitative and quantitative sugar analyses were performed with high-performance thin-layer chromatography as described by Fell (1990) and modified by Campbell et and composition in 1990.

${ }^{2}$ Data shown are the mean of five observations. ration by Tukey's HSD. al. (1990). Sucrose, glucose, and fructose levels were characterized because these are the predominant sugars found in apple nectar (Baker and Baker, 1983; Wykes, 1952). Sorbitol, the major translocated carbohydrate in apple, was not found among a range of apple cultivar nectars in a previous study (Campbell et al., 1990).

Data were tested by analysis of variance and significance levels for the main effects and interactions are presented in Table 1 (only significant interactions). Where there was a significant interaction $(\mathrm{P}>\mathrm{F}=0.05)$, means were separated by Tukey's HSD.

Nectar volume (1.7 to $1.8 \mathrm{pal}$ ), sucrose (55\% to $56 \%$ ), glucose (19\% to $20 \%$ ), and fructose $(25 \%)$ percentages, and sucrose : hexose ratio (1.2 to 1.3 ) were unaffected by either canopy position or defoliation of individual 'Stayman' spurs in 1989 (detailed data not shown). Nectar sugar concentration was $10 \%$ higher in defoliated than in foliated spurs. There was no interaction between canopy position and foliation treatments. Sucrose ( $41 \%$ to $48 \%$ ), glucose (25\% to $30 \%)$, and fructose (25\% to $33 \%)$ percentage > and sucrose : hexose ratio $(0.7$ to 0.9) of 'Delicious' nectar (in 1990) were unaffected by canopy position, girdling, or defoliation of individual spurs (data not shown). Nectar volume was $29 \%$ greater at interior canopy positions than exterior positions, and there was a trend $(\mathrm{P}>\mathrm{F}=0.15)$ for greater sugar concentration at the exterior positions (Table 1). Girdling spurs lowered nectar volume by $92 \%$. With nongirdled spurs, defoliation had no effect on nectar sugar concentration, but with girdled spurs, defoliation lowered sugar concentration by $24 \%$.

The nectar sugar concentration of nongirdled spurs ranged from 500 to $600 \mu \mathrm{g} \cdot \mu \mathrm{l}^{-1}$ ( $50 \%$ to $60 \%$ total sugar), as previously reported for apple nectar (Vansell, 1934; Wykes, 1952). 'Stayman' and 'Delicious' nectar composition was within the range previously reported for apple cultivar nectars (Campbell et al., 1990). Greater evaporation at the exterior positions of 'Delicious' may have caused the reduction in nectar volume, which is supported by the trend for increased sugar concentrations at these positions. Van-

Table 1. The influence of canopy position, defoliation, and girdling on 'Delicious' nectar production

\begin{tabular}{|c|c|c|c|c|}
\hline \multirow{2}{*}{\multicolumn{3}{|c|}{ Treatment }} & \multicolumn{2}{|c|}{ Nectar production } \\
\hline & & & \multirow{2}{*}{$\begin{array}{c}\text { Nectar volume } \\
(\mu l)\end{array}$} & \multirow{2}{*}{$\begin{array}{c}\text { Total sugar concn } \\
\left(\mu g^{*} \mu l^{-1}\right)\end{array}$} \\
\hline Position & Foliation & Girdle & & \\
\hline Interior & \multirow[t]{2}{*}{ Defoliated } & No & $1.39^{z}$ & $572 \mathrm{a}$ \\
\hline Exterior & & No & 1.06 & 596 a \\
\hline Interior & \multirow[t]{2}{*}{ Foliated } & No & 1.28 & 574 a \\
\hline Exterior & & No & 1.05 & $606 \mathrm{a}$ \\
\hline Interior & \multirow[t]{2}{*}{ Defoliated } & Yes & 0.12 & $418 c$ \\
\hline Exterior & & Yes & 0.07 & $428 \mathrm{c}$ \\
\hline Interior & \multirow[t]{2}{*}{ Foliated } & Yes & 0.15 & $524 \mathrm{~b}$ \\
\hline Exterior & & Yes & 0.09 & 592 a \\
\hline \multicolumn{5}{|c|}{ Significance $^{\mathrm{y}}(P>\mathrm{F})$} \\
\hline Foliation (F) & & & 0.562 & 0.036 \\
\hline Girdle $(\mathrm{G})$ & & & 0.001 & 0.012 \\
\hline$F \times G$ & & & 0.244 & 0.037 \\
\hline Position & & & 0.025 & 0.150 \\
\hline
\end{tabular}

Nonsignificant $(\mathrm{P}>\mathrm{F}=0.05)$ interactions are not presented. If interaction significant, mean sepa- 
sell (1934) reported that plum nectar volume and sugar concentration varied greatly during the day due to changing relative humidity, temperature, and wind speed. Nectar volume was unaffected by canopy position in 'Stayman', however, indicating a difference between cultivars or years. Honeybee visitation was not evaluated in our study, but based on previous studies (Wailer, 1972; Wykes, 1952) we doubt that the differences in nectar production and composition measured in our study would influence honeybee foraging behavior within the canopy.

Wykes (1952) reported that girdling and defoliation treatments, applied alone and in combination to flowering shoots of horse chestnut (Aesculus hippocastanum L.), reduced nectar volume, with cessation of nectar production 11 days after girdling and defoliation. In our study, girdling alone also resulted in almost complete cessation of nectar production 7 days after treatment, but defoliation of individual spurs alone had no effect. Girdled and defoliated flowering shoots of horse chestnut had a $38 \%$ lower nectar sugar concentration than the control shoots 5 days after treatment (Wykes, 1952), a result similar to ours with apple. However, in contrast to our results, defoliation of nongirdled horse chestnut shoots also reduced nectar sugar concentration (24\%).

Horse chestnut trees bloom later in the spring than apple and have a large, fully expanded leaf area present at flowering; apple trees have sparse foliage present at flowering. Thus, reserve carbohydrate levels at the time of flowering could be quite different in these two species, possibly influencing the effect of defoliation of spurs or individual shoots on nectar production. Therefore, unlike chestnut, apple nectar production does not depend on the presence of spur leaves, but rather on translocated materials from other sources.

\section{Literature Cited}

Baker, H.G. and I. Baker. 1983. Floral nectar sugar constituents in relation to pollinator type, p. 117-141. In: C.E. Jones and R.J. Little (eds.). Handbook of experimental pollination biology. Van Nostrand Reinhold, New York.

Byers, R. E., J.A. Barden, R.F. Polomski, R.W. Young, and D.H. Carbaugh. 1990. Apple thinning by photosynthetic inhibition. J. Amer. Soc. Hort. Sci. 115:14-19.

Campbell, R. J., R.D. Fell, and R.P. Marini. 1990. Characterization of apple nectar sugars in selected commercial and crab apple cultivars. Fruit Var. J. 44:136-141.

Fell, R.D. 1990. The qualitative and quantitative analysis of insect hemolymph sugars by high performance thin-layer chromatography. Comparative Biochem. Phys. 95A:539-544.

Jackson, J.E. and J.W. Palmer. 1977. Effects of shade on the growth and cropping of apple trees. 11. Effects on componcms of yield. J. Hort. Sci. 52:253-266.

Vansell, G.H. 1934. Relation between the nectar concentration in fruit blossoms and visits of honeybees. J. Econ. Ent. 27:943-945.

Waller, G.D. 1972. Evaluating responses of honeybees to sugar solutions using an artificial-flower feedcr. Ann. Ent. Soc. Amer. 65:857-862.

Wykes, G.R. 1952. The influence of variations in the supply of carbohydrate on the process of nectar secretion. New Phytologist 51:294-300. 